

\title{
ANALISI DELLA CITTÀ BRASILIANA E METODOLOGIE DI RIQUALIFICAZIONE URBANA
}

\author{
ANALYSIS OFTHE CITY AND METHODOLOGIES FOR \\ URBAN REGENERATION
}

Luca Lengueglia

\begin{abstract}
This paper, resulting from the work carried out by students during the Summer Course held in Belo Horizonte, aims to identify, in a clear and concise way, regions of the Brazilian city, where two large categories can be perceived: the formal and the informal city. Subsequently, it examines the best urban development methodologies and what they comprise.
\end{abstract}

Key words: Formal city; Informal city; Urban development. 


\section{La città informale o favela}

La favela, come ambiente socio-urbano, nasce alla fine degli anni '70 per crescere continuativamente fino ai nostri giorni, con alcuni differenti caratteri. Se i primi abitanti di queste regioni urbane erano parte della popolazione rurale che, sotto il miraggio della prosperità economica, si erano inurbate in cerca di fortuna, oggi la percentuale maggiore del tasso di crescita della popolazione che abita la favela è data dal suo incremento di natalità e solo in minima parte da apporti esterni dovuti, ad esempio, dall'immigrazione campagna-città. II problema però è rimasto lo stesso ed è, a fronte di un aumento della popolazione, quella della cronica mancanza di abitazioni che porta le persone ad accettare condizioni di vita del tutto inadeguate.

Questa situazione è stata sempre considerata come un problema fino a quando, nel 1983, non si è riusciti ad inquadrare la questione sotto un altro punto di vista. Anziché vedere la favela come un luogo in cui esistevano, ed esistono, problemi di ordine igienico/sanitario, sociale e criminale e quindi, per estensione, un problema essa stessa, si osservò che di fatto la favela non era altro che un luogo di associazione urbana nato spontaneamente come risposta a determinate necessità, prima fra tutte la casa.

Ecco dunque che la favela da problema è divenuta soluzione, o meglio, I'inizio della soluzione del problema dell'abitazione che ogni uomo o donna sente come primario nell'arco della sua vita.

Questo cambiamento non è solo stato un esercizio di logica o filosofia, ma si è concretizzato con l'emanazione della legge 3532 del 1983 o legge Pro-Favela. Questo è stato un passo importante perché per la prima volta la favela è stata riconosciuta come parte integrante della città e soprattutto, ha consentito alle famiglie che occupavano un territorio illegalmente per costruire la propria dimora di poter continuare ad occupare i lotti e, tramite un procedimento legale, ottenere la proprietà degli stessi. Con questo cambiamento di politica si è passati dal "dover essere espulsi" al "diritto di restare". Ciò ha avuto due conseguenze importanti: la prima per il fatto che, divenendo proprietarie, le persone hanno iniziato ad investire nella loro casa, con conseguente miglioramento delle condizioni di vita, la seconda è stato il crearsi di una posizione culturale a partire dalla quale le persone si sono assunte la responsabilità di provvedere a se stesse ed alle proprie famiglie dando inizio ad un processo risolutivo.

Infine la favela è stata definita dal punto di vista urbanistico come "città informale" poiché normalmente edificata su terreni occupati abusivamente o non dichiarati edificabili per ragioni geologico-ambientali, in contrapposizione alla "città formale" costituita da quella parte di territorio urbano "legale" sia dal punto di vista giuridico che urbanistico secondo le norme del piano regolatore.

\section{La città formale}

È importante chiarire che la favela non è né una città, né un organismo autosufficiente che vive adiacente o separato dal 
resto dell'ambiente urbano considerando, come è evidente, che gli abitanti della favela hanno contatti quotidiani con la città formale, basti pensare ad esempio alla massa di lavoratori che attraversa le varie parti della città per compiere il tragitto casalavoro.

Questo stretto legame fa si che le problematiche che affliggono la città informale abbiano conseguenze rilevanti anche sulla città formale. La criminalità ad esempio, generata nelle aree informali della città, può estendere la sua influenza su una vasta parte del territorio urbano creando un ambiente insicuro e pericoloso che da una parte presenta residenze precarie e inadeguate perché prive, ad esempio, dell'allaccio alla rete fognaria o alla rete idrica e dall'altra, abitazioni o gruppi di abitazioni dotate di tutti i comfort ed i servizi possibili, di fatto isolate e chiuse dal contesto sociale circostante per motivi di sicurezza. In tal modo l'insicurezza creata dai problemi sociali di una parte importante della città ne condiziona pesantemente l'altra parte.

Se quindi si considera la casa come quell'ambiente che garantisce riparo dall'esterno, sicurezza e comfort, ma che, allo stesso tempo, permette dialogo e scambio con l'esterno si può osservare che, usando un paradosso, la città brasiliana può essere ritenuta priva di case che rispondono a questi requisiti. Nella città informale non si hanno case, perché in quelle aree le abitazioni non garantiscono né sicurezza né comfort e dall'altra parte, nella città formale, le abitazioni non "permettono di dialogare" e di creare relazioni sociali con il resto della città.

L'ambiente urbano è dunque caratterizzato da abitazioni che non sono case, ma una sorta di rifugio dove cercare sicurezza.

È evidente che il problema dello sviluppo nella città brasiliana non è circoscritto alla città informale, ma si estende a tutto l'ambiente urbano. Seguendo questo ragionamento si potrebbe pensare che risanando la città informale si avrebbe come conseguenza immediata il miglioramento della sicurezza nella città formale; d'altronde se il problema della sicurezza è dovuto alla criminalità della città informale, causata a sua volta dalla misere condizioni di vita, allora, migliorando le condizioni di vita ed eliminando la criminalità si avrebbe un incremento di sicurezza nella città formale. Questo ragionamento tuttavia appare piuttosto semplicistico. La situazione che si è analizzata, non contempla solo problemi di carattere sociale, giuridico ed urbanistico, ma ha anche problemi di carattere umano e di correlazione tra le varie parti della città, soprattutto tra i cittadini di diverse classi sociali ed etnie. Un ulteriore problema allo sviluppo della città brasiliana che emerge è perciò l'integrazione che non va intesa solo tra città formale e città informale, ma in tutte le sue componenti sociali ed economiche.

La città deve essere quell'ambiente che permette la convivenza di persone diverse, che si confrontano su un piano egualitario sfruttando mutuamente le risorse che ciascuno può offrire, con il fine comune di migliorare, sotto l'aspetto sociale, economico, urbanistico e legislativo, la propria comunità. La città brasiliana non ha quindi ancora raggiunto pienamente l'obiettivo di essere comunità integrata. 


\section{Processi di riqualificazione e sviluppo urbano}

Benché i concetti fin qui elaborati siano stati appresi in un particolare contesto che presenta problemi specifici di natura sociologica ed urbana, come si è visto nei paragrafi precedenti, non sembra tuttavia immotivato considerare tali aspetti nei diversi ambiti urbani informali, siano essi a nord o a sud dell'equatore, si chiamino "slum" o "loop" e siano visibili, come sempre più spesso accade, anche nei Paesi cosiddetti sviluppati.

Quando I'approccio ai problemi esposti poc'anzi è quello di un processo di riqualificazione $\mathrm{o}$, più in generale, un intervento urbanistico le esperienze di successo più significative condotte in vari contesti hanno messo in evidenza pochi, ma essenziali aspetti. In primo luogo occorre ricercare una soluzione duratura e condivisa dalle parti in causa al fine di evitare un probabile insuccesso. Qualsiasi intervento deve interpretare le esigenze dei vari attori coinvolti, che possono in prima approssimazione essere distinti in coloro che vivono la città e coloro che la usano; vanno considerati diversi aspetti come quello ambientale ed economico e, inoltre, si deve tenere conto delle risorse a disposizione quasi sempre limitate.

La prima condizione per realizzare un intervento di riqualificazione duraturo è quella della costruzione di un processo partecipativo, il quale consiste in un lavoro di concerto tra le amministrazioni pubbliche e gli "stakeholders" in modo che si possano comporre interessi e conflitti. Per far ciò è necessario possedere alcune elementari e rare qualità come la capacità di ascolto, di apprendere e costruire, bisogna avere un forte senso di responsabilità e le scelte che si operano devono essere fatte in relazione alle risorse disponibili.

L'intervento partecipativo è una soluzione procedurale che coinvolge i destinatari dell'opera di riqualificazione per far in modo che possano essere comprese a fondo le necessità dei beneficiari dell'aiuto e soprattutto che cosa questi si aspettino dall'intervento. Un processo di questo tipo, dunque, deve definire, oltre agli obiettivi, anche il ruolo della comunità e lascia tuttora dei problemi irrisolti come, ad esempio, il recupero dei costi, la sicurezza del diritto di proprietà e il problema dello "scaling up".

Statisticamente si è visto che, nell'ambito di interventi volti a costruire case popolari per potervi alloggiare persone che non possono affrontare il costo di un alloggio, la percentuale di coloro che rivendono il bene immobile per tornare nei luoghi da cui sono stati rimossi è del $35 \%$ mentre quando si è eseguita la stessa operazione attraverso un processo partecipativo, questa percentuale è scesa sotto il $5 \%$. Ciò sembra identificare chiaramente il percorso da seguire per arrivare alla soluzione migliore ed in modo altrettanto chiaro la motivazione che spiega questa differenza. II metodo partecipativo coinvolge e responsabilizza la comunità coinvolta nel processo, rendendola partecipe di un risultato ottenuto non solo da "altri", ma di cui essa stessa diventa protagonista propositiva in quanto portatrice delle proprie particolari necessità da soddisfare. 
Questo è il motivo che sembra garantire al metodo partecipativo così alte probabilità di successo, ma che lo rende allo stesso tempo di difficile attuazione. II fatto che si basi principalmente sull'individuazione di necessità particolari di comunità specifiche lo fa divenire difficilmente ripetibile, né assicura una rapida attuazione. È necessario costruire, di volta in volta, un rapporto di reciproca fiducia tra chi riceve l'aiuto, o è coinvolto nell'intervento di riqualificazione, e l'operatore dell'azione di risanamento. In generale possono essere individuati per tali interventi di riqualificazione urbana alcuni soggetti. II primo, come appare evidente, è la comunità che necessita dell'intervento di riqualificazione, la quale da "oggetto" dell'aiuto nei processi "non partecipativi" diviene soggetto principale dell'azione con quello "partecipativo". Il secondo è il promotore dell'intervento, generalmente identificato nella pubblica amministrazione. Inoltre spesso le difficili situazioni da affrontare richiedono finanziamenti così ingenti da rendere necessario l'intervento di una terza figura in qualità di finanziatore esterno; questi a sua volta richiede che l'operazione rispetti certi requisiti ritenuti essenziali per la buona riuscita dell'impresa. Infine, ma spesso di fondamentale importanza, al processo partecipa un ulteriore soggetto che riveste un ruolo di intermediario tra il soggetto promotore e la comunità. In generale tale ruolo è ricoperto da un'organizzazione non governativa (ONG) che si fa portavoce degli interessi della comunità. Talvolta una di queste figure può incarnare più soggetti, come nel caso di amministrazioni pubbliche particolarmente forti o per interventi di poco rilievo economico, nel qual caso il promotore dell'intervento diviene anche finanziatore dello stesso.

Il processo di riqualificazione ha successo quando tutti i soggetti coinvolti riescono a collaborare finalizzando ogni energia e risorsa sull'obiettivo finale, ma questo non basta a concluderlo in modo del tutto soddisfacente. Lo scopo ultimo di un intervento di questo genere, infatti, non equivale al solo recupero urbanistico dell'area ma attraverso programmi economici e sociali paralleli ad uno sviluppo armonico ed integrato di quella zona nel contesto della città.

A tal fine è necessario sensibilizzare la comunità, attraverso un costruttivo dialogo che rafforzi la consapevolezza delle proprie capacità e la volontà di operare. II ruolo delle ONG può quindi essere anche quello di formare la comunità non tanto e non solo sugli aspetti tecnici urbanistici ed edilizi ma anche per favorire lo sviluppo integrale della persona valorizzando quel capitale umano, che diversamente rimarrebbe inespresso. Questa azione di formazione andrebbe quindi integrata in qualsiasi azione di risanamento perché, una volta raggiunto l'obiettivo primario e risanata l'area il processo, possa continuare ad evolvere e a durare nel tempo. Attraverso questa azione di formazione parallela a quella di riqualificazione fisica della città la persona e la comunità potrà decidere autonomamente di se stessa e del proprio futuro e soprattutto sarà in grado di poter comprendere e non subire passivamente avvenimenti anche esterni che possano condizionare negativamente il proprio sviluppo.

Ciò può migliorare sensibilmente il processo di riqualificazione urbana e consentire di raggiungere obiettivi soddisfacenti, condivisi e duraturi. La città brasiliana non ha quindi ancora raggiunto pienamente l'obiettivo di essere UNA comunità integrata. 


\section{Riferimenti}

ARAÚJO, Marinella Machado. Usucapião coletivo: possibilidades e desafios. Belo Horizonte, PUC Minas, Summer School 2008.

BASTOS, Paulo. Urbanização de favelas. Estudos Avançados, São Paulo, v. 17, n. 47, p. 212-221, jan./abr. 2003.

FOLLONI, Giuseppe. L'educazione è il motore dello sviluppo. Belo Horizonte, PUC Minas, Summer School 2008.

FOLLONI, Giuseppe. Slum upgrading: un approccio integrato e partecipato: il caso di Ribeira Azul. Belo Horizonte, PUC Minas, Summer School 2008.

IMPARATO, Ivo. Brasil: políticas para favelas e assentamentos informais. Belo Horizonte, PUC Minas, Summer School 2008.

MINAS GERAIS. Lei n. 3.532, de 06 de Janeiro de 1983. Autoriza o executivo municipal a criar o programa municipal de regularização de favelas - Profavela e dá outras providências. Minas Gerais, Belo Horizonte, 6 de Janeiro de 1983

MONTI, Carlo. Pianificazione sostenibile e qualità del progetto urbano. Belo Horizonte, PUC Minas, Summer School 2008.

SENN, Lanfranco. Le condizioni per la sostenibilità dello sviluppo urbano. Belo Horizonte, PUC Minas, Summer School 2008.

\section{Indirizzo}

Luca Lengueglia

Via Bisentolo Sud, 535

40014 Crevalcore (BO) - Italy

lucalengueglia@gmail.com 